\title{
Angular Gyrus Involvement at Encoding and Retrieval Is Associated with Durable But Less Specific Memories
}

\author{
Marieke van der Linden, ${ }^{1}$ ㅈoud M.W.J. Berkers, ${ }^{1}{ }^{\circledR}$ Richard G.M. Morris, ${ }^{2}$ and Guillén Fernández ${ }^{1}$ \\ ${ }^{1}$ Donders Institute for Brain, Cognition and Behaviour, Radboud University Nijmegen Medical Centre, $6500 \mathrm{HB}$ Nijmegen, The Netherlands, and ${ }^{2} \mathrm{Centre}$ for \\ Cognitive and Neural Systems, The University of Edinburgh, EH8 9JZ Edinburgh, United Kingdom
}

\begin{abstract}
After consolidation, information belonging to a mental schema is better remembered, but such memory can be less specific when it comes to details. A neuronal mechanism consistent with this behavioral pattern could result from a dynamic interaction that entails mediation by a specific cortical network with associated hippocampal disengagement. We now report that, in male and female adult human subjects, encoding and later consolidation of a series of objects embedded in a semantic schema was associated with a buildup of activity in the angular gyrus $(\mathrm{AG})$ that predicted memory $24 \mathrm{~h}$ later. In parallel, the posterior hippocampus became less involved as schema objects were encoded successively. Hippocampal disengagement was related to an increase in falsely remembering objects that were not presented at encoding. During both encoding and retrieval, the AG and lateral occipital complex (LOC) became functionally connected and this interaction was beneficial for successful retrieval. Therefore, a network including the AG and LOC enhances the overnight retention of schema-related memories and their simultaneous detachment from the hippocampus reduces the specificity of the memory.
\end{abstract}

Key words: angular gyrus; fMRI; hippocampus; memory; schema

\section{Significance Statement}

This study provides the first empirical evidence on how the hippocampus and the neocortex interact dynamically when acquiring and then effectively retaining durable knowledge that is associated to preexisting knowledge, but they do so at the cost of memory specificity. This interaction is a fundamental mnemonic operation that has thus far been largely overlooked in memory research.

\section{Introduction}

In time, most of the details of our experiences are forgotten. However, some information is retained for a longer period of time and thought to be stored in neocortical networks that are separate from the hippocampus (Scoville and Milner, 1957; Squire, 1986; Bontempi et al., 1999). This selectivity of memory retention is the basis of the standard model of system-level consolidation (Alvarez and Squire, 1994; Frankland and Bontempi, 2005). After consolidation, the medial prefrontal cortex (mPFC) and midline cortical regions have been observed to be activated during memory retrieval in rodents (Bontempi et al., 1999; Frankland et al., 2004; Maviel et al., 2004; Takehara-Nishiuchi et al., 2006; Takehara-Nishiuchi and McNaughton, 2008; Goshen et

Received Nov. 23, 2016; revised Aug. 12, 2017; accepted Aug. 17, 2017.

Author contributions: M.v.d.L., R.M.W.J.B., R.G.M.M., and G.F. designed research; M.v.d.L. performed research; M.v.d.L. analyzed data; M.v.d.L., R.M.W.J.B., R.G.M.M., and G.F. wrote the paper.

This work was supported by the European Research Council (Advanced Grant 268800 to G.F. and R.M.) R.B. is currently affiliated with the Max Planck Institute for Human Cognitive and Brain Sciences, Leipzig, Germany. M.v.d.L. is currently affiliated with Máxima Medisch Centrum, Veldhoven, The Netherlands. We thank Hester Breman for programming the sdmcalculator plug-in for BrainVoyager.

The authors declare no competing financial interests.

Correspondence should be addressed to Dr. Marieke van der Linden, Donders Institute for Brain Cognition and Behaviour, Centre for Cognitive Neuroimaging, P.0. Box 9101, NL-6500 HB Nijmegen, The Netherlands. E-mail: marieke.vanderlinden@gmail.com.

DOI:10.1523/JNEUROSCI.3603-16.2017

Copyright $\odot 2017$ the authors $\quad 0270-6474 / 17 / 379474-12 \$ 15.00 / 0$ al., 2011) and humans (Takashima et al., 2006; Gais et al., 2007; Takashima et al., 2009; Bonnici et al., 2012).

Schemas provide a "fast track" into successful consolidation. Schemas are frameworks of acquired knowledge that are implemented in the brain as networks of interconnected neocortical representations (Wang and Morris, 2010). Schemas facilitate the assimilation of related new information, leading to better retention (Bransford and Johnson, 1972; Tse et al., 2007; van Kesteren et al., $2010 \mathrm{~b})$. The mPFC is more involved in processing memories congruent with a schema compared with schema-incongruent memories (van Kesteren et al., 2010b; Tse et al., 2011; van Kesteren et al., 2013b; van Kesteren et al., 2014; Brod et al., 2015). Initial evidence suggests that the parietal cortex also participates in applying a schema to an experimental task (Hanson et al., 2007; Sweegers et al., 2014; van Buuren et al., 2014).

This study investigates the possibility that the angular gyrus (AG) plays a key role in binding sensory content into a schema. Within the ventral parietal cortex, AG is optimally located at the junction of visual, spatial, somatosensory, and auditory processing streams. These sensory-motor attributes all converge in the AG, where the perceptual details are believed to be abstracted (Fernandino et al., 2016) and bound together by semantic and conceptual associations (Binder et al., 2009). After consolidation, the AG recombines schema components into a single memory representation (Wagner et al., 2015). 


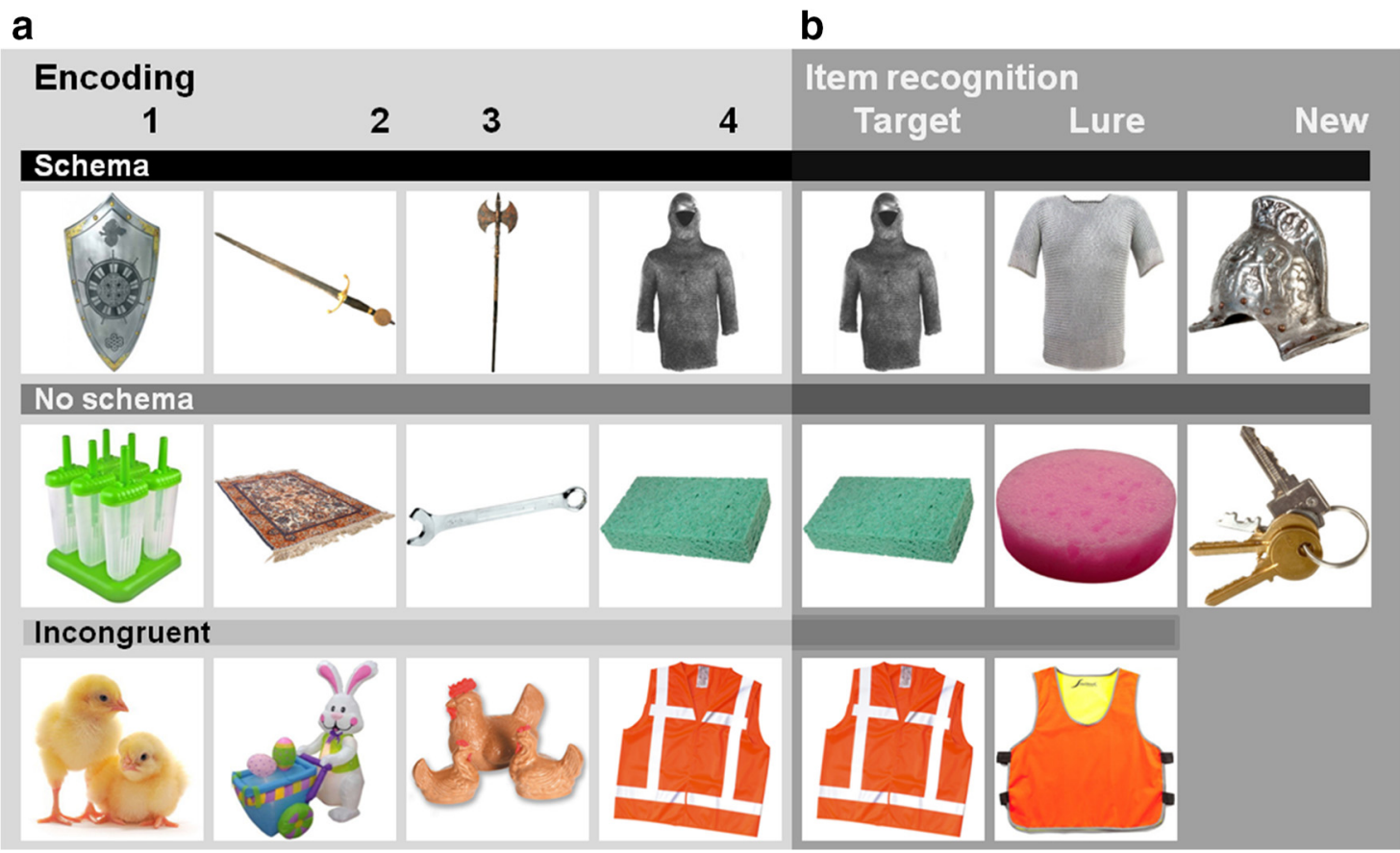

Figure 1. Stimuli. $a$, During both encoding sessions, participants were presented with quartets belonging to the schema, no-schema, and incongruent conditions. A quartet consisted of four objects that were presented serially. The first quartet (row 1) is an example of the "knight" schema. Next, in the no-schema condition (row 2), four objects that have no preexisting association were presented. For the incongruent condition (row 3), the first three objects from the "Easter" schema were followed by an incongruent final object (e.g., a high-visibility vest). $\boldsymbol{b}$, During item recognition, the participants were presented with the quartets' final objects (column 1). The quartet's final object was presented once exactly as seen before (target) and once as a very similar exemplar of the same object (lure; column 2). In addition (column 3), there were objects that were new and belonged to the schemas that were presented (new schema related) or new but unrelated objects (new schema unrelated).

We sought to elucidate the roles of the medial temporal lobe (MTL), mPFC, and AG in the encoding and consolidation of new information, followed by the later retrieval of recent (withinsession) and remote (24 h earlier) schema-associated memories. During encoding, a series of four object photographs were presented in sequence (Figs. 1, 2). In the schema condition, these objects were all related to a real-world semantic schema (e.g., horse, spurs, boots, and a cowboy hat). If the AG were involved in processing schema-related objects, then successive presentation of these objects would modulate activity in the AG in a different way than a series of semantically unrelated objects. At the same time, the buildup of a schema could lead to disengagement of the hippocampus during encoding. An additional condition was included in which the last object of the set was incongruent with the schema (e.g., a Christmas tree instead of the cowboy hat). We expected the AG to respond differently to the final object based on its incongruence with the preceding object set. Finally, we also considered the implications of schemas with respect to subsequent retrieval. Shimamura (2011) suggested that the AG links "episodic features with long-term memory networks," an idea that leads to the prediction that such networks (schemas) will be recruited as the schema is built up. This dynamic process could in turn influence the encoding and/or consolidation of schemarelated information and the AG should differentiate between subsequent retrieval of objects that were embedded in a schema during encoding compared with objects that were not.

The neocortical regions that likely support lasting memory representations include brain areas involved in the original processing of the stimulus at encoding (Tulving and Thomson, 1973; Nyberg et al., 2000; Danker and Anderson, 2010). In the case of visually presented objects, these include ventral visual areas such as the lateral occipital complex (LOC). To test our prediction that the AG would be functionally connected to visual representation areas during encoding and item recognition and that successful linking to these object representations would be beneficial to memory retention, we included a functional localizer scan.

\section{Materials and Methods}

\section{Participants}

In total, 31 participants participated in the experiment. For seven participants, the data were excluded from the final analyses for the following reasons: two participants did not complete the study, one participant moved $12 \mathrm{~mm}$ during scanning, one participant fell asleep during encoding on the second day, and three participants displayed memory performance that did not exceed chance level. The presented results stem from a dataset with 24 right-handed participants ( 2 males, 22 females, mean age: 23.5 years, range $18-30$ years). All participants were neurologically healthy and were paid for their participation (10 euros/h with an additional 2 euros for every $10 \%$ that they scored above $50 \%$, i.e., chance level). For two of 24 participants, the datasets were incomplete because of scanner malfunction during one of the encoding sessions. For these incomplete datasets, the data were adjusted in further analyses (i.e., the objects they did not see during encoding were removed from the item recognition test, maximum of $20 \%$ of the trials).

\section{Stimuli}

Stimuli consisted of color photographs of objects. These photographs were taken from the Hemera Object Database and a Google image search. Objects were shown on a white background and were made to fit exactly in a box of $300 \times 300$ pixels while keeping their aspect ratios intact.

In our experiment, a schema was defined as a group of objects that are all related to each other through a semantic theme. More specifically, a schema consisted of four pictures of objects (a quartet) from the same theme (see Fig. $1 a$ for an example of a "knight" schema). In total, we used 100 themes consisting of places, characters, seasons, sports, events, holidays, professions, rooms, countries, etc. The schemas were created by selecting sets of four objects that ostensibly fitted within a theme based on 
a separate preparatory study $(n=20)$. Participants were presented with a theme written on a screen and were instructed to type in the names of at least 10 objects that they associated with this theme. The nine objects that were mentioned most frequently were used to form two quartets per schema and one related new object (schema-based lure). Schema strength of an object was identified as the proportion of participants who mentioned an object within a given theme. When creating the quartets, the schema strength of the objects was taken into account by being divided equally over positions in the quartets (on average, $27.5 \%$ for each of the four positions). A second type of quartet (incongruent) was constructed by shuffling the Schema quartets' final objects around so that this object did not fit with the other three objects in the quartet anymore (Fig. $1 a)$. We also constructed 100 no-schema quartets in which there was no a priori association between the objects (for an example, see Fig. 1a). The schema consistency of the schemas and the absence of schema for the no-schema quartets was confirmed in a second preparatory study $(n=20)$. A different group of participants were asked to press a button during serial presentation of the objects in the quartets as soon as the schema was known to them. For the no-schema quartets, there were no reports of schemas by the participants, whereas, for the schema quartets, the average number of participants that correctly reported the schema was $90 \%(15 \% \mathrm{SD})$.

\section{Image acquisition}

During encoding and retrieval, whole-brain images (T2*-weighted multi-echoplanar imaging, 39 slices, $2 \mathrm{~mm}$ thick with $0.5 \mathrm{~mm}$ gap, repetition time $=2190 \mathrm{~ms}$, in-plane resolution $=2.5 \times$ $2.5 \mathrm{~mm}^{2}, 4$ echo times: TE1 $=9.4 \mathrm{~ms}, \mathrm{TE} 2=21.9 \mathrm{~ms}$, TE3 $=34 \mathrm{~ms}$, $\mathrm{TE} 4=47 \mathrm{~ms}$, flip angle $=90^{\circ}$, field of view $=21.2 \mathrm{~cm}$ ) were acquired on a 3 T whole-body MR scanner (MAGNETOM Skyra; Siemens). Fat saturation was turned off. During the functional localizer scan, we acquired whole-brain images (echoplanar imaging, 45 slices, $2 \mathrm{~mm}$ thick with 0.5 $\mathrm{mm}$ gap, repetition time $=2390 \mathrm{~ms}$, in-plane resolution $=2.5 \times 2.5$ $\mathrm{mm}^{2}, \mathrm{TE}=30 \mathrm{~ms}$, flip angle $=90^{\circ}$, field of view $=21.2 \mathrm{~cm}$ ). In addition, a high-resolution structural T1-weighted 3D magnetization prepared rapid acquisition (MPRAGE) gradient echo sequence image was obtained after the functional scan (192 slices, voxel size $=1 \times 1 \times 1 \mathrm{~mm})$.

\section{Experimental design and statistical analysis}

Design and procedure. Participants were scanned on 2 consecutive days (Fig. 2a). On the first day, they encoded (for $53 \mathrm{~min}$ ) the first set of objects (called the "remote" condition by virtue of its distance from retrieval the next day). Before they started with this first encoding session, they were scanned using a functional object localizer. After $24 \mathrm{~h}$ (sleep duration was on average $7.9 \mathrm{~h}$ with an SD of $45 \mathrm{~min}$ ), they returned to the laboratory for the second encoding session ("recent" condition, 53 $\mathrm{min}$ ). After the second encoding session, they had a short break outside of the scanner and then returned inside the scanner for the item recognition memory task probing memory for both remote and recent items (58 min).

Functional localizer. An independent functional localizer was included to allow us to investigate at a later time point whether psychophysiological interaction (PPI) connectivity maps from memory areas overlapped with visual representation areas that respond preferentially to objects. The participants were told what the purpose of the localizer scan was and that they need not memorize the pictures that they were shown. We used a block design with 32 photographs of common objects (unrelated to the schemas in the main experiment) and 32 scrambled pictures from a standard functional localizer task to localize the LOC (Malach et al.,
1995). Images had gray backgrounds and measured $500 \times 500$ pixels and were assigned randomly to blocks of 17 images (each image was displayed for $400 \mathrm{~ms}$, followed by a blank screen of $600 \mathrm{~ms}$ ). Each block lasted $16.4 \mathrm{~s}$. Within each block, 16 images were unique and one image was repeated. The participants were instructed to detect this repetition by pressing a button with the index finger of the right hand. Each block was followed by a blank screen interval of $10 \mathrm{~s}$. Each image was presented twice to the subject, but within different blocks. Four blocks of objects and four blocks of scrambled objects were presented. The localizer run lasted $3.5 \mathrm{~min}$.

Encoding. During each of two memory encoding sessions (called "remote" and "recent" in chronological order of presentation), 150 quartets were serially presented to the participants, one object after the other (Figs. 1a, 2b). Quartets belonged to three schema conditions: (1) the schema condition, in which all objects in the quartet belong to one theme; (2) the incongruent condition, in which the final item of the quartet was incongruent with the theme provided by the first three items; and (3) the no-schema condition, in which there was no obvious association (schema) between the objects. The participants were instructed to remember all objects and quartets. They were also told that, during the final memory test, they would be presented with very similar lures so that they should try to remember as many details as possible. Simultaneously, they were asked to indicate for each object whether it would fit inside a shoebox. They responded "yes" or "no" with the index or middle finger of their right hand. Participants were told that memorizing the objects and quartets had priority over the "shoebox task." A black fixation cross was visible on screen throughout the encoding session and changed to red to signal the start of a new quartet. The red fixation cross was presented for $500 \mathrm{~ms}$. The objects were presented for $1500 \mathrm{~ms}$ and the interstimulus interval was $3.5 \mathrm{~s}$ on average. All interstimulus intervals were jittered between 1.5 and $5.5 \mathrm{~s}$. During each encoding session, they were presented with 600 objects, so, across the two encoding sessions, 
participants memorized 300 quartets containing 1200 objects. We installed a few safeguards to minimize memory to specific items (that arise from some objects being more memorable than others): First, the quartets were counterbalanced between subjects over remote and recent encoding sessions. Second, targets and lures were counterbalanced over subjects. Third, the quartet's final objects were semicounterbalanced over schema conditions because the objects in the fourth position of each quartet could not be swapped between the schema and no-schema quartets. Therefore, final objects were counterbalanced over schema and incongruent conditions across subjects and a second counterbalancing was performed in terms of the assignment of objects to the incongruent and no-schema conditions.

Retrieval. Retrieval was tested by an item recognition memory task. The experiment started with three practice trials with objects that were new and not seen before to familiarize the participant with the timing of the events and the task. During the item recognition task, the participants were presented sequentially with photographs of objects in a pseudorandom order. The quartets' final items were presented as old targets (Fig. 1b). A perceptually similar lure was included for each target (Fig. 1b). Half of the targets were presented before the lure and half afterward. Moreover, the spacing in time between a target and its lure was maximized. In addition, 50 objects that were related to a schema, but that were not presented during encoding, served as new schema objects during retrieval (Fig. 1b). There were another 50 objects that were new and not related to the presented schemas, the new no-schema objects. This resulted in a total of 700 objects that were presented to the participants (see Fig. $2 c$ for an overview of all conditions).

After the presentation of a fixation cross $(500 \mathrm{~ms})$, each object was presented for $1500 \mathrm{~ms}$ (Fig. 2b). Subjects rated their memory for the objects using a confidence scale ranging from 1 (very surely old) to 6 (very surely new). Half of the participants responded "old" with their index, middle, and ring finger of their right hand and responded "new" with their index, middle, and ring finger of the left hand and this was reversed in the other half of the participants. After a jittered interval (average $=3.5 \mathrm{~s}$, range: $1.5-4.5 \mathrm{~s}$ ), during which the confidence levels were displayed on screen, the next object was presented.

After the retrieval session, the participants filled in a short questionnaire asking them about strategies used during encoding (90\% reported using the schemas during encoding) and retrieval (17\% reported using the schemas during retrieval) and the amount of sleep on the previous night (on average, $8 \mathrm{~h}$ ).

Behavioral data analyses. For each subject, the proportion of hits ("old" responses to old objects), misses ("new" response to old objects), correct rejections ("new" response to new objects and similar lures), false alarms ("old" response to new objects and similar lures), and "no responses" were calculated per condition, as well as the confidence levels and response times for each of these variables. Trials were included at all levels of confidence because there was above chance memory performance at all confidence levels. To account for response bias, we subtracted the proportion of false alarms made when presented with the new no-schema objects from, respectively, the proportions of schema, noschema, and incongruent hits (to targets) and false alarms (to lures). Proportion of hits minus false alarms and response times were tested in two (separate) multivariate analyses of variance (MANOVAs) with two factors: schema condition (with three levels: schema, incongruent, and no-schema) and study-test delay (with two levels: remote and recent). Additional differences between conditions were tested using pairedsamples and one-sample $t$ tests. All reported $p$-values are two-tailed. Alpha was set to 0.05 .

MRI data analyses. The multiecho fMRI data were processed using in-house software written in MATLAB version 7.5 (The Mathworks), which used the first 29 scans of the session (during which the experiment had not yet commenced) to calculate the optimal weighting of echo images for each voxel (i.e., by using a weighted measure of the contrastto-noise ratio for each echo/scan). Motion correction was performed with reference to the first echo and the realignment parameters were applied to the other echoes using iterative rigid body realignment. Next, calculations of optimal echo weight for each voxel were used to combine multiecho fMRI data into single images. The combined images were further processed using BrainVoyager QX (Brain Innovation). Images were slice-time corrected (using sinc interpolation). Coregistration of functional and 3D structural measurements was computed by relating T2*-weighted images and the T1-weighted MPRAGE measurement, which yields a $4 \mathrm{D}$ functional dataset. Structural $3 \mathrm{D}$ and functional $4 \mathrm{D}$ datasets were transformed into Talairach space (Talairach and Tournoux, 1988) and spatially smoothed with a Gaussian kernel (FWHM $=8 \mathrm{~mm}$ ).

The expected BOLD signal change was modeled using a gamma function (tau of $2.5 \mathrm{~s}$ and a delta of 1.5) and convolved with each presented object (Boynton et al., 1996). Regressors were time locked to the onset and duration of the presentation of the objects. Data were corrected for serial correlation using the AR2 method and a percentage signal change transformation was performed before statistical analysis. Statistical analyses were performed using the general linear model. For the encoding sessions, we modeled the three conditions (schema, incongruent, and no-schema) separately and subsequently remembered or forgotten separately. This means that all objects in a quartet were categorized as to whether the final object was remembered or forgotten as follows: Condition1_Object1_Remembered, Condition1_Object2_Remembered, Condition1_Object3_Remembered, and Condition1_Object4_Remembered. In the encoding sessions, the contrasts included only the final objects from the quartets. For the contrast between remembered and forgotten items, the items that were forgotten were collapsed across schema conditions. This ensured more trials in the forgotten conditions and we presume the same process for forgetting in all conditions (e.g., schema remembered $>$ schema forgotten + no-schema forgotten + incongruent forgotten).

We also constructed a parametric model in which the 4 objects within a block were given parametric weights $(-1.5,-0.5,0.5$, and 1.5$)$ and, per condition, the blocks were also divided depending on whether the final objects were subsequently remembered or forgotten. For the retrieval session, we modeled the first three trials (not uniquely part of any condition: "warm-up" trials), hits, misses, false alarms, and correct rejections separately for schema, incongruent, and no-schema remote and recent objects and to new no-schema and new schema objects for a total of 29 regressors. Random-effects group analyses were performed using ANOVA to test for interactions. The first analysis consisted of a randomeffects ANOVA with three factors: schema condition (schema, noschema, incongruent), study-test delay (recent, remote), and memory (hits, misses).

In addition, in all models, six derivatives of the realignment parameters were included as well as regressors for spikes related to motion (one regressor per spike). Furthermore, $t$ tests were used to test contrasts between different conditions. Results were thresholded at the voxel level at $p<0.001$ and corrected using Monte Carlo cluster threshold correction completed $>1000$ iterations. Small volume correction was applied to the ventro-mPFC using false discovery rate correction on the voxels that were included in an anatomical mask of Brodmann areas 32 and 24. Significantly activated clusters in $\mathrm{MPFC}$ and AG were selected for a more sensitive region of interest (ROI) analysis. For the ROI analyses, the $\beta$ values were extracted from all voxels within the ROI and averaged over ROI, subject, and condition. The ROI time courses were standardized so that $\beta$-weights (regression coefficients) of predictors, as indices of effect size, reflect the BOLD response amplitude of one condition relative to the variability of the signal. $\beta$-weights were obtained for all voxels within these ROIs per subject and per condition. Differences between the subject-averaged $\beta$-weights were investigated by paired $t$ tests with a threshold set at $p<0.05$. All $t$ tests were two-tailed.

Finally, functional connectivity analyses (PPI) were conducted to determine the interactions between physiological data in terms of the psychological processes of the experiment (Friston et al., 1997). PPI methodology followed the steps first described in Friston et al. (1997) and was implemented using a BrainVoyager plugin (sdmcalculator version 0.2). For the PPI analysis on the retrieval data, the seed region that we used was the AG region that was found in the schema $>$ no-schema contrast during retrieval. The time course was extracted and averaged across all voxels from this area for each subject. As the psychological regressor, we used the contrast schema remote hits $(+1)$ and no-schema remote hits $(-1)$. For a second PPI analysis using the left parietal ROI, we used the same methodology. As the seed, we used the areas found in the 
a

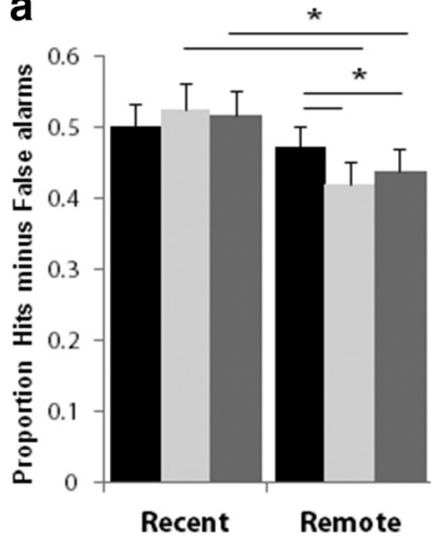

b

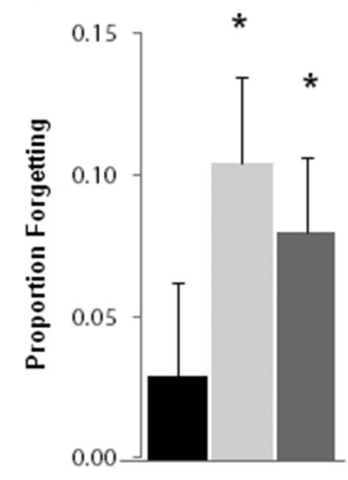

C

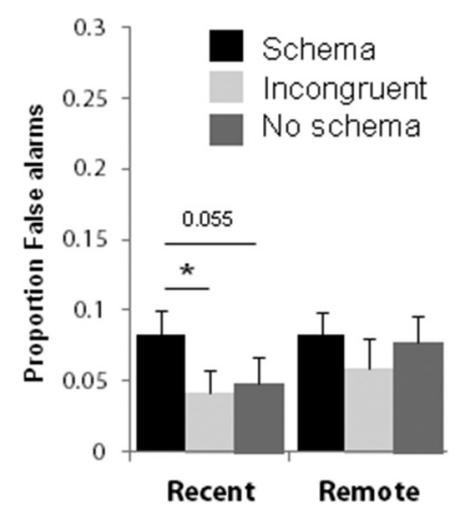

d New objects

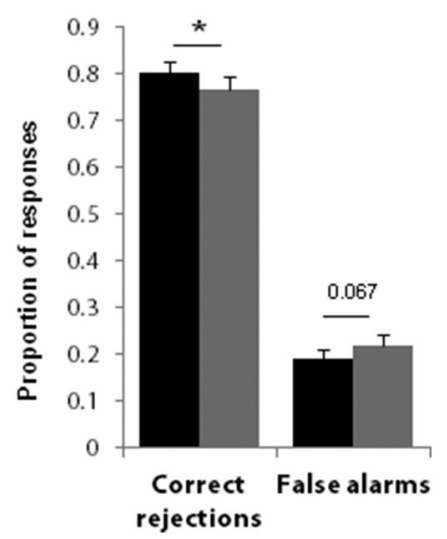

Figure 3. Behavioral data. $\boldsymbol{a}$, Proportion of hits (hits minus false alarms to the new no-schema condition) are presented for recent and remote conditions for the schema, incongruent, and no-schema conditions. $\boldsymbol{b}$, Proportion of forgetting (recent minus remote hits) is presented for the three schema conditions. $\boldsymbol{c}$, Proportion of false alarms for all schema conditions (false alarms minus the proportion of false alarms to the new no-schema condition) are presented for recent and remote conditions. $\boldsymbol{d}$, Proportion of correct rejections and false alarms to the new no-schema and new schema objects are presented. For all plots, black color represents the schema condition, dark gray incongruent, and light gray no schema. Error bars indicate SEM. ${ }^{*} p<0.05$.

contrast schema $>$ no-schema and extracted per subject the time course from all voxels. The psychological contrast was the same contrast as used for the previous PPI. To test for a general effect of correct responses on connectivity to the LOC, we also calculated a PPI for remote no-schema hits $(+1)$ versus recent no-schema hits $(-1)$. For the PPIs on the encoding data, we used as a seed the AG region that was active in the remote $($ schema $>$ no-schema) $>$ recent (schema $>$ no-schema) and the LOC region from the functional localizer that was active for objects $>$ scrambled.

To investigate the behavioral relevance of the connectivity between the seed and target areas, we performed a correlation analysis at the group level (using Pearson's $r$ ) on the extracted PPI $\beta$-values from the ROI with the behavioral measures: remote schema hits, remote schema effect (schema minus no-schema), and remote schema false alarms. We also calculated correlation with behavioral measures (hits minus the false alarms to new no-schema objects) and the schema buildup in the AG. Schema buildup was measured by subtracting $\beta$-values to object four minus object two (the moment at which a schema can first be detected). The difference between two correlations was tested using software from Lee and Preacher (2013), available online.

\section{Results}

Behavioral data

An important property of schema-based memories is that there is a behavioral benefit; that is, better memory retention for schema-related memories compared with no-schema memories. Although this schema effect can be apparent immediately for associative memory, other studies have found that the difference between schema and non-schema-based item recognition is largest after a period of consolidation (van Kesteren et al., 2013a; Durrant et al., 2015). Our data are consistent with the latter pattern (Fig. 3a). For all schema conditions (schema, no-schema, and incongruent), we calculated recognition performance as hits minus false alarms (where false alarms pertain to the new noschema objects). Participants performed well above chance in all three conditions on both study test delays (all $t_{(23)}>4.9$, $p<0.0001)$. However, there was a significant study test delay by schema condition interaction $\left(F_{(2,22)}=3.67, p=0.04\right.$, Wilk's $\Lambda=0.75)$. There was no difference between schema conditions for recently studied objects (schema $>$ no-schema: $t_{(23)}=-0.93$, $p=0.36$; schema $>$ incongruent: $\left.t_{(23)}=-1.37, p=0.18\right)$. However, for objects encoded the day before, participants had better memory for information embedded in a schema quartet than for either objects encoded in the no-schema condition $\left(t_{(23)}=2.18\right.$, $p=0.04)$ or incongruent quartets $\left(t_{(23)}=2.51, p=0.02\right)$. Memory did not decline over $24 \mathrm{~h}$ for schema objects $\left(t_{(23)}=0.86, p=\right.$ $0.4)$. In contrast, for no-schema $\left(t_{(23)}=3.01, p=0.006\right)$ and incongruent objects $\left(t_{(23)}=3.44, p=0.002\right)$, memory was poorer for remote than for recent objects. Response times for hits also decreased as a result of Study test delay $\left(F_{(1,23)}=23.03, p<0.001\right.$, Wilk's $\Lambda=0.50)$, but there was no difference between schema conditions on this measure. Given this comparison between recent and remote memories, the schema benefit that we found on remote memory scores reflected better retention for schemabased memories (Fig. 3b).

To investigate whether schema-based memories are less specific and more gist-based (Winocur et al., 2010), we included similar lures for each target during retrieval and as new objects. Lures consisted of very similar exemplars of the final object of each quartet that had been presented during encoding (Fig. 1b). If memory for objects within a schema is less specific, then more false alarms would occur to the similar lures compared with other novel objects. Indeed, across conditions, there were more false alarms to the similar lures than to new objects. The key finding was that false alarm rates were highest to the lures of the schema objects that were encoded just before (recent condition: schema $>$ no-schema: $t_{(23)}=2.02, p=0.055$; schema $>$ incongruent: $t_{(22)}=2.21, p=0.038$; Fig. $3 c$ ). However, this pattern of results could also be explained by guesses informed by prior knowledge (i.e., when not sure about seeing the cowboy hat, a participant might be more likely to press "old" because he/she remembered seeing items from a cowboy schema and therefore saying "old" to the cowboy hat increases the chance of a hit). Therefore, we also included objects in the item recognition test that were not seen during encoding, but were congruent with the schemas used during encoding. We observed that these new "schema-related objects" did not elicit more false alarms than the new "schema-unrelated objects." If anything, there was a trend toward the opposite result (no-schema $>$ schema: $t_{(23)}=1.92$, $p=0.067$ ), with the subjects showing more correct rejections to the new schema-related objects than the new schema-unrelated objects $\left(t_{(23)}=2.307, p=0.03\right.$; Fig. $\left.3 d\right)$. This latter finding indicates that the schema is actually beneficial to the correct identification of new related objects as incorrect lures. To conclude, the 


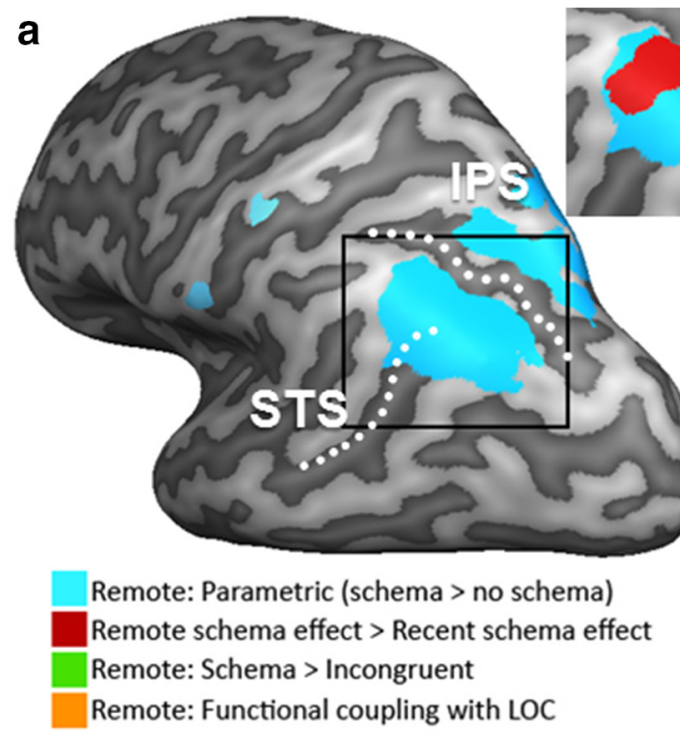

C

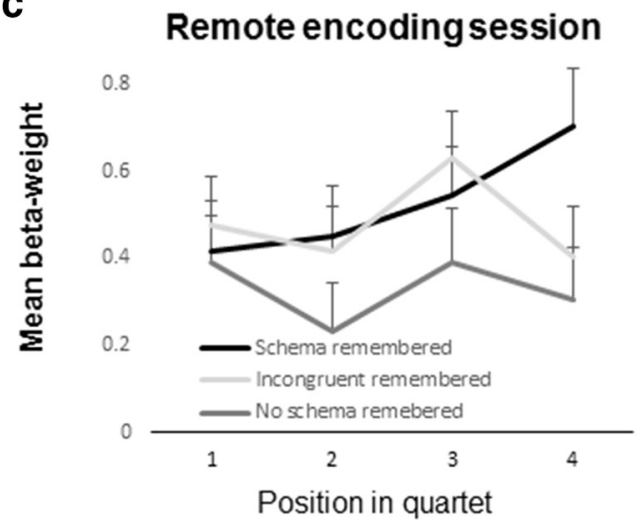

d

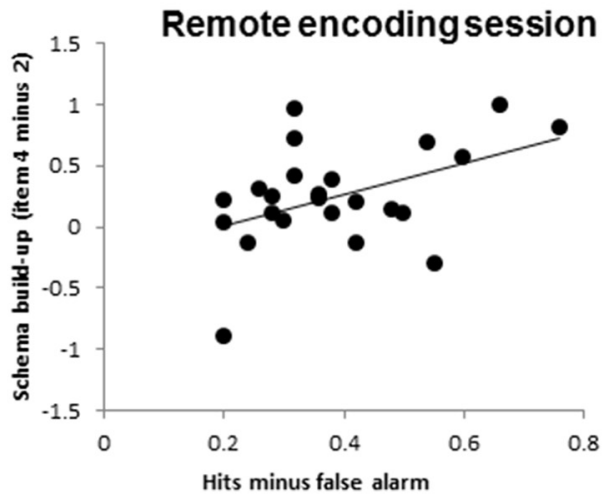

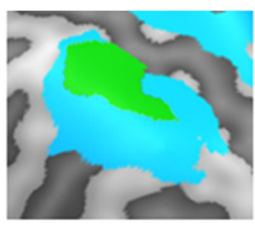

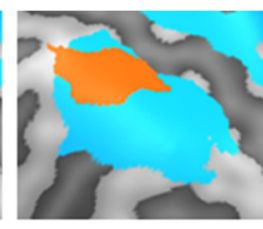

b

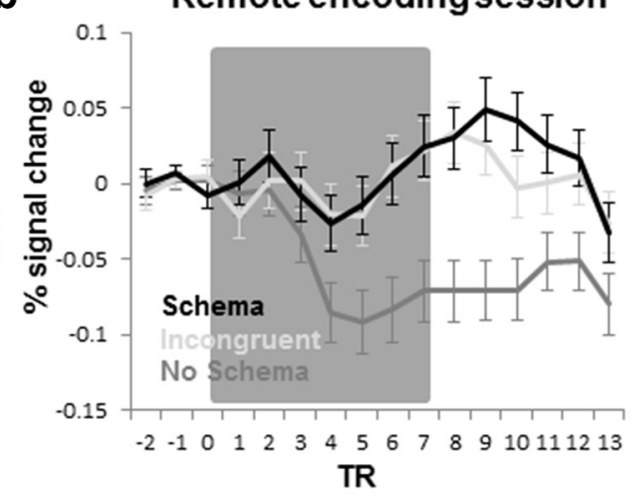

Recent encoding session

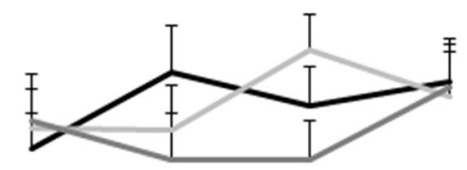

1

2

3

4

Position in quartet

Recent encoding session

$\bullet$
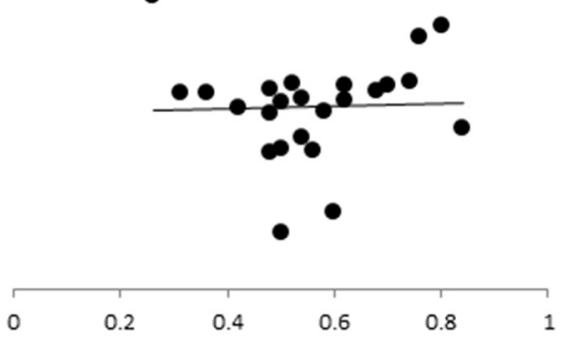

Hits minus false alarm

Figure 4. Schema effects in the AG during encoding. $\boldsymbol{a}$, Inflated hemisphere (left hemisphere, dark gray areas are sulci) with overlays of four contrasts that all converge in the AG. In green are the areas that were more active in the remote encoding session to schema than to incongruent remembered final objects. In orange are areas that showed functional coupling with the $L O C$ during encoding of subsequently remembered schema objects. In red are areas that showed a larger schema effect in the remote encoding session compared with the recent encoding session as follows: (remote schema remembered $>$ remote no-schema remembered) $>$ (recent schema remembered $>$ no-schema remembered). In blue are areas that showed a parametric schema effect; that is, a buildup of activation with each object presentation (parametrically modulated activity during schema quartets $>$ parametrically modulated activity during no-schema quartets). The dotted lines present the superior temporal sulcus (STS) and intraparietal sulcus (IPS) and are depicted for anatomical reference. All maps were corrected at a cluster level of $p<0.05$ (voxel-level threshold was $p<0.001$ ). $\boldsymbol{b}$, Subject-averaged event-related time course from the AG region defined by the contrast: remote (schema $>$ no-schema) $>$ recent (schema $>$ no-schema) and presented by the red area in $\boldsymbol{a}$. We plotted the percentage signal change at each TR ( $2190 \mathrm{~ms})$ for the threeschema conditions (black: schema, light gray:incongruent, dark gray: no schema). The gray area indicates the time of the presentation of the quartet; the first objectis presented at TR $=0$ and the offset of the final object of the quartet is on average (with jittered intervals) at TR $=7$. Error bars indicate SEM. $c$, For each encoding session, we plotted the subject-averaged $\beta$-weight for each of the four objects in the quartet from the same ROl as used in $\boldsymbol{b}$. Schema quartets are in black, incongruent quartets are in light gray, and no-schema quartets are in dark gray. $\boldsymbol{d}$, Correlation between the buildup of a schema ( $\beta$-weight to object 4 minus $\beta$-weight to object 2 ) and the proportion of hits minus false alarms is plotted for both encoding sessions (from the same ROl used in $\boldsymbol{b}$ ). The remote encoding session was the session $24 \mathrm{~h}$ before retrieval. The recent encoding session was the session immediately preceding retrieval.

supposition that the schema is used for informed guessing can be ruled out. To the contrary, objects that were embedded in a schema during encoding have less specific but more resilient memory traces.
To summarize, we have shown that, shortly after encoding, the memory for schema objects was already less specific, leading to more false alarms to lures from recently seen schema objects and this difference between false alarm rates disappeared after a 
delay of $24 \mathrm{~h}$ In addition, we found a behavioral benefit for schema objects after overnight consolidation. Schema objects showed no decay in item recognition memory performance, whereas the no-schema and incongruent objects did display forgetting. We conclude that schema memories were less specific immediately after encoding, but were retained better over $24 \mathrm{~h}$.

\section{fMRI data}

Encoding

Neural correlates of subsequent schema memory. The behavioral data demonstrated that, after a short delay, recognition was at ceiling. The difference in memory performance between schema conditions became apparent after $24 \mathrm{~h}$, and was reflected in better retention of schema-embedded objects. Therefore, the analysis of subsequent memory effects here relates to the delay by schema interaction found in memory performance. To identify brain regions specifically involved in retention, $t$ test comparisons were performed comparing the subject-averaged " $\beta$-maps" from the schema contrasts between the remote and recent encoding sessions using the contrast (remote schema remembered $>$ remote no-schema remembered) $>$ (recent schema remembered $>$ no-schema remembered). We found that the AG showed a schema $>$ no-schema difference that was larger for remote than for recent encoding (Fig. $4 a$, red overlay). For the separate contrast of schema $>$ incongruent over time (remote vs recent encoding), we did not find any region at conventionally corrected thresholds, but an uncorrected voxel threshold of 0.005 did point to differential activation in the AG, hippocampus, superior frontal, and cingulate gyrus.

We plotted the event-related subject-averaged time course and the subject-average $\beta$-weights from the AG region that displayed a larger schema effect with consolidation (Fig. $4 b, c)$. For all conditions, activation started at a comparable level, but then progressively differed as the schema unfolded. Activation built up monotonically for both the schema and incongruent quartets, but this effect was not seen for the no-schema quartets. Importantly, activity dropped for the incongruent condition when the final object no longer fitted the previously built-up schema. Because the AG region was defined based on the remote (schema $>$ no-schema) $>$ recent (schema $>$ no-schema) contrast, we did not perform inferential statistics comparing the extracted signal across conditions.

To test whether this buildup of a schema is predictive of better performance at retention, we calculated the amount of activity in the schema that was buildup (from the first moment the schema can be inferred at object position 2, up to the final fourth object of the quartet). This measure of schema buildup in the AG in individual participants during encoding correlated with $24 \mathrm{~h}$ retention measured as schema hits minus false alarms, the latter pertaining to the new no-schema lures) of those same participants $(r=0.43, p=0.036)$. This correlation implies that the buildup of schema activity in the AG is beneficial for retaining object memories over $24 \mathrm{~h}$ This effect was specific to objects encoded in the first; that is, the "remote encoding session." In the recent encoding session, there was no schema buildup that was specific for the subsequently remembered objects and no positive correlation between a possible buildup (the difference in activity to object 4 minus object 2) with successful memory retrieval scores $(r=0.04, p=0.85)$. We also tested whether the activity decrease for the fourth object (response to the fourth object minus response to the third object) in the incongruent quartets would be indicative of memory performance (hits minus false alarms to new no-schema objects) because this object might stand out more, similar to an oddball, but we found no evidence for this $(r=-0.8, p=0.71)$.

\section{Table 1. Schema buildup}

\begin{tabular}{lrrrr}
\hline Area & \multicolumn{1}{c}{$x$} & $y$ & \multicolumn{1}{c}{$z$} & \multicolumn{1}{c}{$t$} \\
\hline Parametric increase & & & & \\
$\quad$ R middle frontal gyrus & 30 & 17 & 31 & 5.40 \\
$\quad$ Thalamus & 12 & -10 & 4 & 4.79 \\
$\quad$ Parahippocampal gyrus & -6 & -61 & 4 & 5.47 \\
$\quad$ Linferior frontal gyrus & -30 & 32 & 19 & 5.56 \\
L angular gyrus & -30 & -67 & 37 & 5.91 \\
$\quad$ L superior temporal gyrus & -36 & -25 & 10 & 4.72 \\
Parametric decrease & & & & \\
$\quad$ R posterior hippocampus & 27 & -40 & 10 & -5.18 \\
\hline
\end{tabular}

Shown are areas modulated by the parametric regressor for schema buildup in the remote encoding session for quartets with a subsequently remembered final object. Coordinates are Talairach coordinates of the peak voxel. For all $t$ values $(\mathrm{df}=23), p<0.0001$

Schema buildup across object sequences. An analytically interesting comparison can be made between the final objects of the schema and incongruent quartets that were remembered after $24 \mathrm{~h}$. In both cases, these objects were preceded by three objects that belonged to a schema, with only the incongruent fourth object being out of place. Importantly, this contrast was significant in the AG (bilaterally) and the right supramarginal gyrus (Fig. $4 a$, green overlay). This means that, whereas a host of regions was preferentially activated for a series of schematically congruent versus incongruent objects, only the AG and supramarginal gyrus were preferentially activated to the fourth object being congruent versus incongruent with the preceding object. This finding points to the $A G$ as the candidate area for holding the schema representation.

The previous analyses already demonstrated (indirectly) a parametric buildup of activity in the AG as successive objects were presented. This buildup is clearly schema related, as demonstrated by the observed difference between the contrasts for schema and no-schema objects and between schema and incongruent. That is, the initial three objects are modulating AG activity buildup for schema quartets that contain the subsequently remembered object. Although we had no reason to expect a similar parametric buildup for the no-schema condition, a general alteration of activity as a consequence of sequential visual input could not be ruled out. However, our data establish that the AG is not a candidate area for such a general effect, but there might be other regions that do show either a parametric buildup or even a parametric decline. Therefore, we also performed whole-brain analyses to identify regions where activity was modulated by a parametric regressor in either encoding session for quartets that contained subsequently remembered objects. The parametric regressor represented the hypothesized buildup or decline of activation with each successive presentation of an object in the quartet containing the remembered final object. We tested for activity that fitted the parametric regressor for the quartets containing the subsequently remembered final objects. An overview of all brain regions is presented in Table 1. In the remote encoding session, the remembered no-schema quartets did not elicit reliable parametric variation in brain activity in any region, whereas the remembered incongruent quartets showed a parametric increase in the left superior frontal gyrus and a decrease in the right AG. As expected, during the remote encoding session, activity for the schema quartets that contained the subsequently remembered objects was parametrically modulated in the AG, confirming the analyses presented above. A particularly interesting observation was a parametric decrease for the remembered schema quartets in the hippocampus (Fig. $5 a$, blue overlay), which is suggestive of a disengagement of the hippocampus when encoding schema memories. 


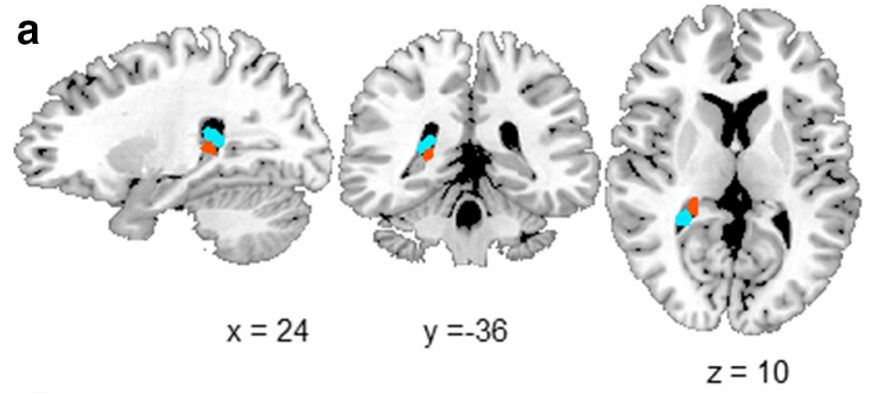

Parametric decrease for Schema quartets Connectivity from the AG

C

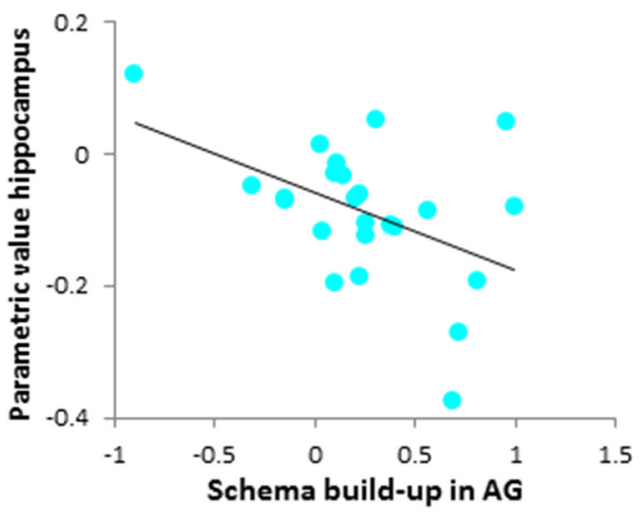

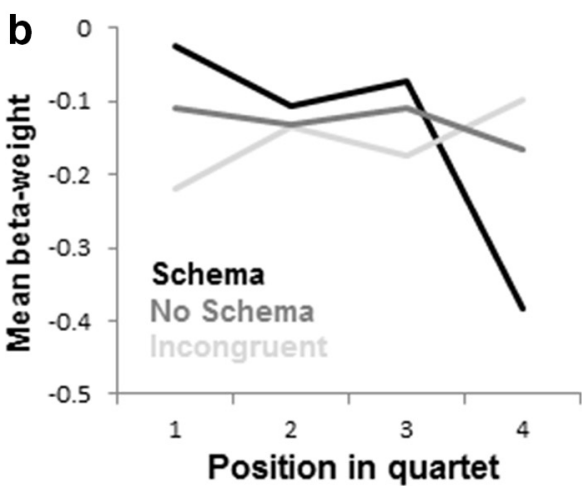

d

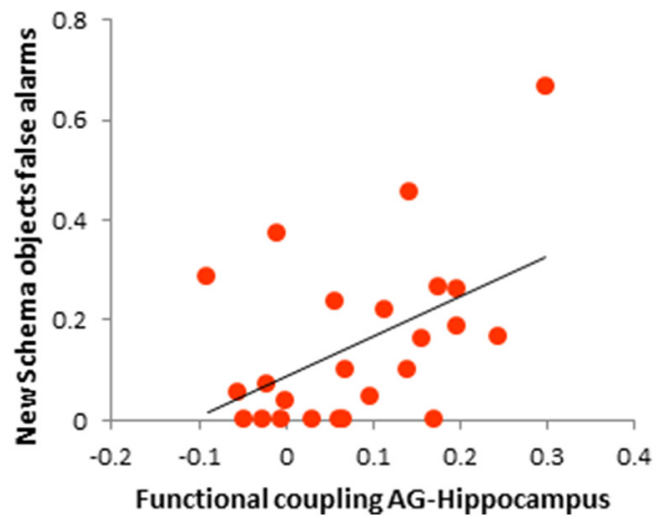

Figure 5. Hippocampal deactivation during encoding of schema quartets. $\boldsymbol{a}$, Blue overlay shows the posterior hippocampus area in which activity decreased parametrically for schema quartets in the remote encoding session. Because the map shown is depicting a group-averaged, normalized, spatially smoothed overlay, voxels extend into the ventricle (6\% overlap with the anatomical mask of the hippocampus). However, the peak voxel of the activation $\left(x=23, y=-37, z=13\right.$, parametric decrease $\left.t_{(23)}=4.398197, p<0.001\right)$ falls within the posterior hippocampus. In red ( $98 \%$ overlap with the anatomical mask of the hippocampus) is the result from the PPI connectivity analysis with the AG as a seed region showing that the posterior hippocampus is modulated by the parametric increase of schema-related activity in the AG. Both maps are thresholded at a cluster-level-corrected $p<0.05$. $\boldsymbol{b}$, Subject-averaged $\beta$-weights from the hippocampal ROI showing the parametric decrease (represented by the blue area in $\boldsymbol{a}$ ) for each of the four objects in the quartet as a function of schema condition. Schema quartets are shown in black, incongruent quartets in light gray, and no-schema quartets in dark gray. c, Subject-averaged $\beta$-values representing the parametric scores of the hippocampal Rol that showed a parametric decrease in activity (represented by the red area in $\boldsymbol{a}$ ) correlated with the amount of schema buildup in the AG. $\boldsymbol{d}$, PPI values from the connectivity analysis from the AG to the hippocampus correlate with the number of false alarms participants made to the new schema objects.

For the recent encoding session, we found no parametric modulation of activity in subsequently remembered schema quartets. When taking remembered and forgotten schema quartets together, the left AG did show a parametric increase in activity (peak: $x=-45, y=-64, z=22, t_{(23)}=3.99, p<0.001$ ). This shows that, for recently encoded objects, the schema is being built up in the AG as well, but that this buildup is apparently only beneficial for remembering these objects after a period of $24 \mathrm{~h}$. This is consistent with the buildup being associated with consolidation. We also found no parametric schema effect for subsequently remembered quartets (schema parametric $>$ no-schema parametric). During remote encoding, we found a parametric schema effect in areas that largely overlapped with the main effect of schema (Fig. $4 a$, blue overlay, Table 2), including the AG.

Because the AG is modulated by the buildup of a schema by presenting related objects, we suspected that the object representations in the ventral visual stream could contribute to the schema buildup. A connectivity analysis (PPI) using the lateral occipital region from the localizer scan (objects $>$ scrambled) as seed did indeed show that this region was connected to the AG during encoding of schema objects (Fig. $4 a$, orange overlay). This $A G$ area overlaps almost completely with the $A G$ regions showing a remote schema effect and schema buildup.

Interplay between $A G$ and hippocampus during encoding of schema quartets. The parametric decrease that we observed in
Table 2. Parametric schema effect

\begin{tabular}{lrrrl}
\hline Area & \multicolumn{1}{l}{$x$} & \multicolumn{1}{c}{$y$} & \multicolumn{1}{c}{$z$} & $t$ \\
\hline R superior temporal gyrus & 57 & -1 & 4 & 4.51 \\
Bilateral parietal and cuneus & 9 & -82 & 25 & 6.63 \\
R precentral gyrus & 33 & -16 & 43 & 4.35 \\
R superior frontal gyrus & 27 & 59 & 19 & 4.50 \\
Precuneus & 15 & -46 & 40 & 5.46 \\
L inferior occipital gyrus & -6 & -61 & 4 & 4.52 \\
L middle frontal gyrus & -30 & 53 & 10 & 5.98 \\
L precentral gyrus & -51 & -22 & 40 & 5.03 \\
L postcentral gyrus & -57 & -22 & 22 & 5.07 \\
\hline
\end{tabular}

Shown are areas with a larger parametric buildup for schema versus no-schema quartets in the remote encoding session. Coordinates are Talairach coordinates of the peak voxel. For all $t$ values ( $\mathrm{df}=23), p<0.001$.

the hippocampus (Fig. $5 b$ ) raises the intriguing possibility that the connection between the AG and hippocampus is inhibitory in nature and leads to the hippocampus becoming less involved in encoding memories when a schema is present. This fits with a model of accelerated systems-level consolidation for schema memories. Interestingly, the parametric value for the schema decrease in the posterior hippocampus correlated negatively with the amount of schema buildup in $A G$ $(r=-0.45, p=0.027$; Fig. $5 c)$. This indicates that a greater schema buildup effect was paired with a stronger parametric decrease of activity in the hippocampus. This could mean that 
the AG, as it is becoming more involved in encoding schema objects, starts signaling the hippocampus that its involvement is no longer necessary. To test this idea, we performed a PPI connectivity analysis with the same AG seed region, investigating increased connectivity as a function of the parametric buildup of a schema. This analysis revealed that the right posterior hippocampus is indeed functionally coupled to the AG during encoding and that the degree of connectivity is modulated in association with the parametric buildup of the schema during presentation of the quartets (Fig. $5 a$, red overlay).

The differential expression of detailed versus gist-like memory representations may reflect differential activation in hippocampus and cortex. Specifically, if the hippocampus disengages, then there may be a less detailed memory trace. We therefore examined the correlation between false alarms and AG-hippocampus PPI connectivity scores. Those participants with higher AG-hippocampus PPI connectivity did not show more false alarms to schema lures ( $r=0.07, p=\mathrm{ns}$ ), but they did show a higher rate of false alarms to new schema objects $(r=0.48, p=0.018)$. The higher number of false alarms to new objects from the same schema may indicate that, when detailed memory of the schema objects is lacking, because of less hippocampal involvement during encoding, the gist of the schema prevails.

\section{Retrieval}

Interaction between schema condition and study test delay in $m P F C$ at retrieval. It was of interest to explore whether the neuronal schema effect during retrieval mirrors the schema effect found during encoding and in the behavioral data, in the sense that they become apparent only after consolidation. An interaction between schema condition and study test delay was observed in the $\mathrm{mPFC}$ during retrieval (coordinates of the peak: $x=-6, y=39$, $z=2, F_{(1,23)}=26, p<0.05$, small volume correction; Fig. 6). To secure insight into the direction of this interaction, we extracted $\beta$-weights from the mPFC for remote and recent hits in each condition. When comparing these subject-averaged $\beta$-weights, we found that $\mathrm{mPFC}$ activity was higher for schema than noschema for remote hits $(t=2.54, p=0.011)$ and schema activation was also higher for remote than recent schema hits $(t=2.33$, $p=0.020)$. However, incongruent hits did not differ from either schema (remote: $t=1.39, p=0.16$ ) and no-schema hits (remote: $t=1.09, p=0.27)$. MPFC activity to incongruent hits showed a trend toward increased activity over time (incongruent: remote $>$ recent: $t=1.87, p=0.06$ ). This interaction confirmed that the schema differences in MPFC become apparent after a $24 \mathrm{~h}$ delay at the same time as the behavioral benefit arises.

Schema effect in parietal cortex. To test whether there were brain areas that were more activated by schema relative to noschema during item recognition, we used a direct contrast between conditions (omitting the misses). We found five areas that responded more to schema than no-schema: the right $A G$, left parahippocampal gyrus, left precuneus (extending into the cuneus), dorsal medial thalamic nucleus, and left inferior parietal lobe (Fig. 6). No areas were more active during retrieval of noschema or incongruent objects than schema objects. Because the AG showed behaviorally relevant schema activation during encoding, the AG and parietal areas were further inspected in an ROI analysis (Fig. 7). Using a PPI analysis with remote schema memories versus remote no-schema memories (hits), we found connectivity with the AG to be higher for the remote schema memories in several areas in the left hemisphere (Fig. 8). Furthermore, we found increased connectivity between AG and LOC that correlated positively with correct recognition (proportion
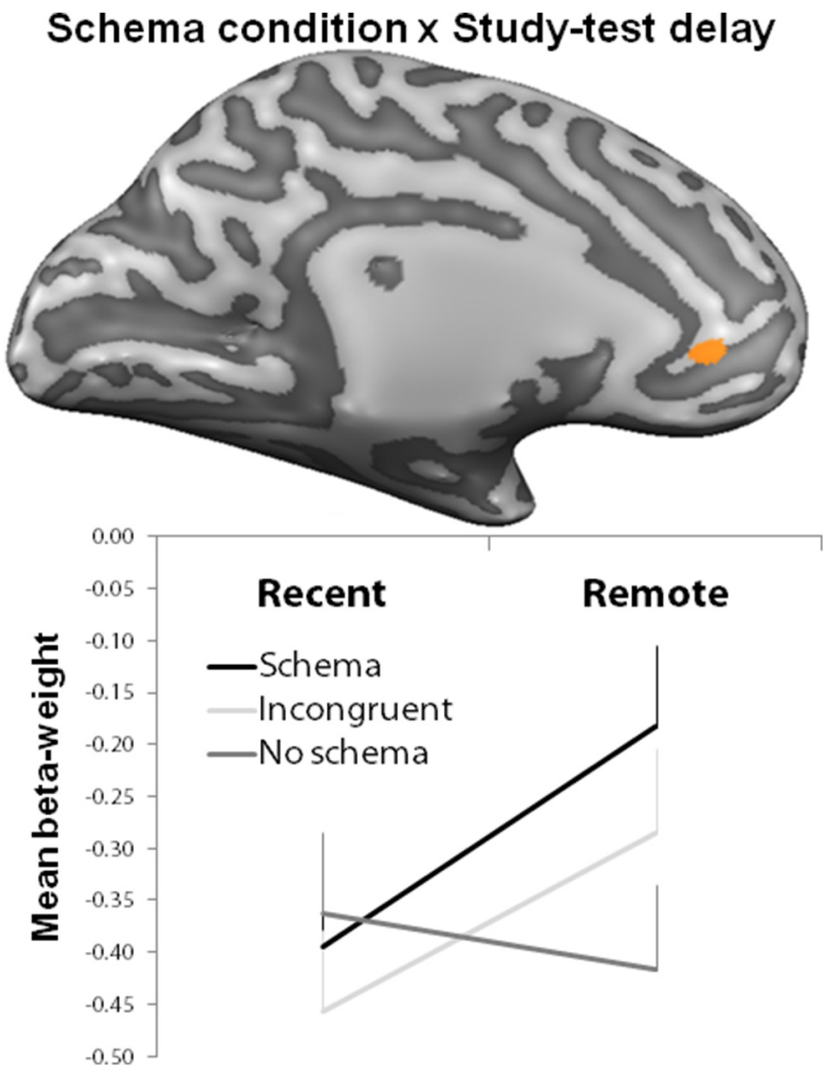

Figure 6. Study-test delay by schema condition interaction during retrieval. We found a study test delay by schema condition interaction in the right mPFC (coordinates of the peak: $x=$ $-6, y=39, z=2, F_{(1,23)}=26, p=0.00004$, SVC corrected at $\left.<0.05\right)$ during retrieval. The $\mathrm{mPFC}$ result is presented on an inflated right hemisphere; dark gray colors represent the sulci. Plotted below are subject-averaged $\beta$-weights extracted from all voxels within this mPFC region for schema (black), no-schema (dark gray), and incongruent (light gray) remote and recent hits to provide information on the direction of the interaction and are shown here for this purpose. Error bars indicate SEM.

hits) of remote schema objects $(r=0.42, p=0.039)$. To rule out a general effect of retrieval success, we also calculated the correlation between the PPI value in the AG and hits to no-schema objects and found no correlation $(r=-0.18, p=\mathrm{ns})$; the difference between both correlations was significant $(Z=2.08, p=$ 0.038 ). These findings suggest that the AG is specifically involved in successful retrieval of schema objects.

\section{Discussion}

Schemas are believed to be beneficial for memory of conceptually related information (Bransford and Johnson, 1972) and are thought to accelerate consolidation (Wang and Morris, 2010; van Kesteren et al., 2012; Ghosh and Gilboa, 2014). Here, we found behavioral and neuroimaging evidence for accelerated consolidation for schema memories that could be linked to memory processes in the AG, hippocampus, and $\mathrm{mPFC}$ during encoding and retrieval.

First, objects embedded in a schema (the schema consisted of the successive presentation of related objects) were better remembered $24 \mathrm{~h}$ later compared with those that were either not embedded in a schema or incongruent with one. Second, during encoding, the AG showed an activity pattern that reflected the buildup of a schema. This buildup of activity was specific for schema memories that were retained for $24 \mathrm{~h}$, with the amount of AG buildup predictive of memory retention. One interesting aspect of "buildup" is that it parallels old data (Bransford, 1979; 

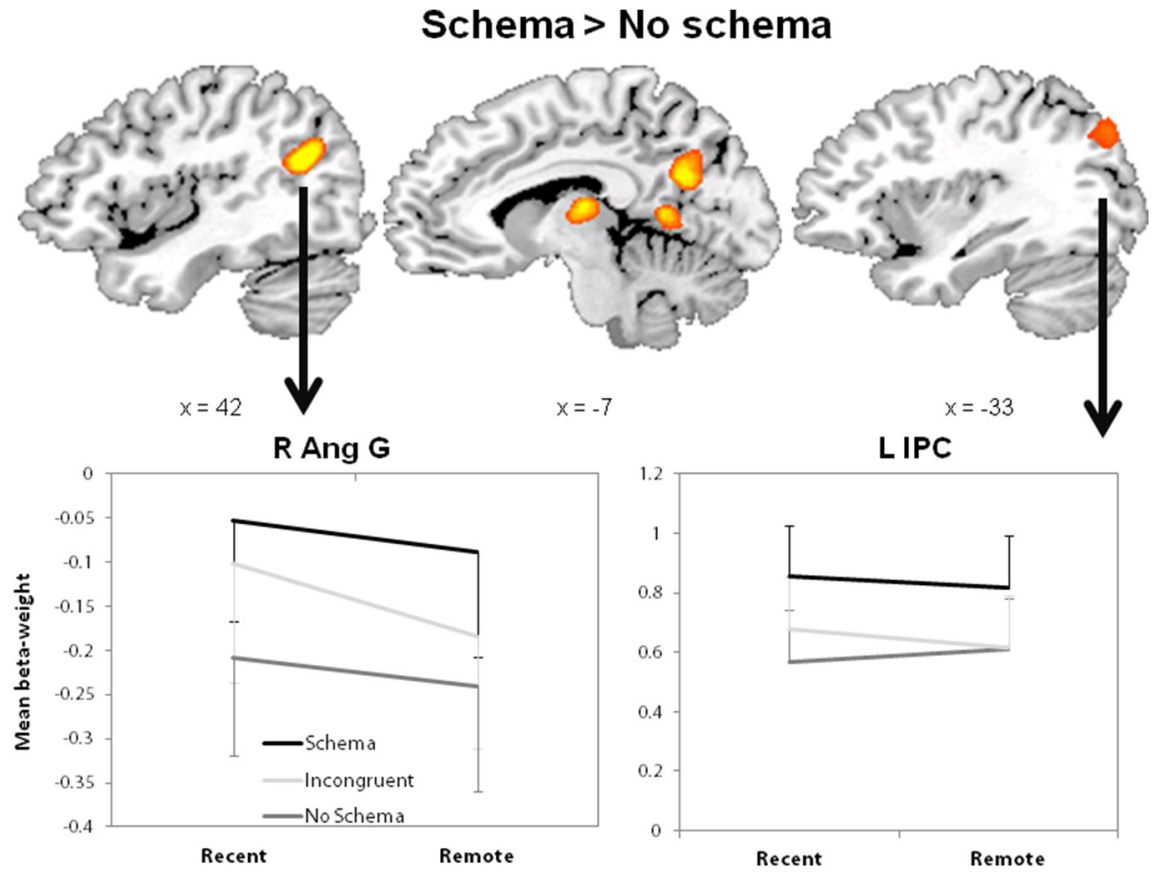

Figure 7. Areas active during schema retrieval. The contrast between schema and no-schema objects (collapsed over time) was displayed as an overlay on three sagittal slices. The graphs below present the $\beta$-weights from both ventral parietal areas. RAng G, Right AG; LIPC, left intraparietal cortex. Error bars indicate SEM.
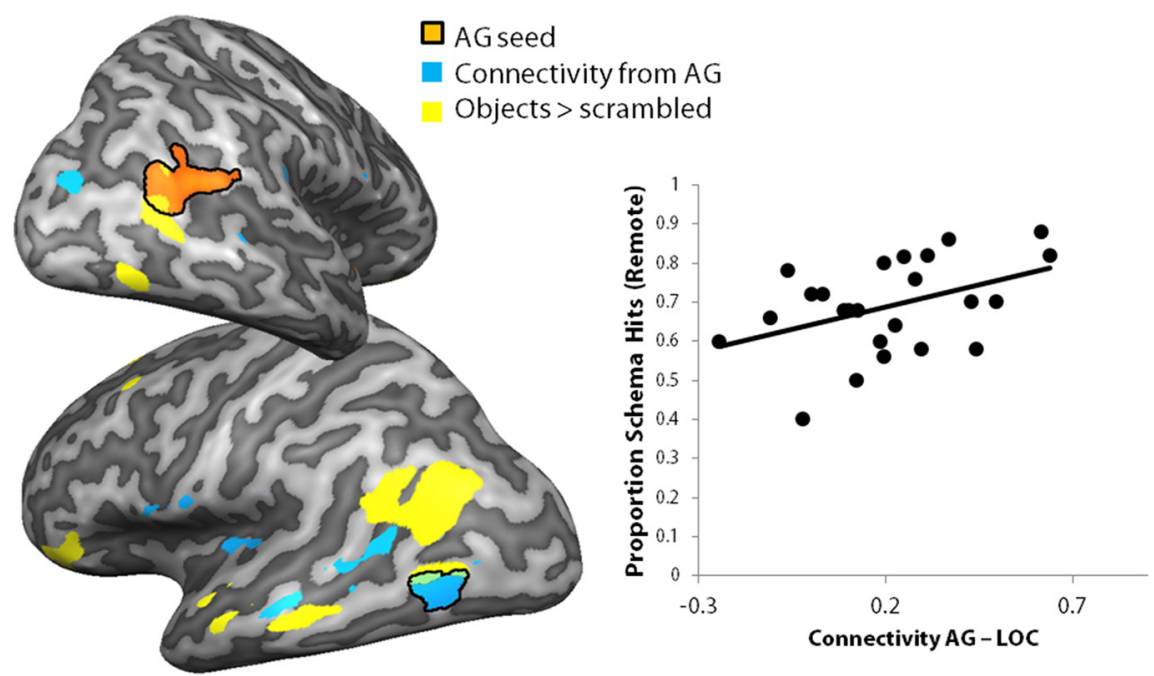

Figure 8. AG connectivity during schema retrieval. Connectivity from the AG seed region (in orange) was explored with a PPI analysis for remote schema objects compared with remote no-schema objects (overlay in blue). These results are overlaid on two inflated hemispheres and combined with the overlay (in yellow) from the functional localizer contrasting objects with scrambled objects. The right graph represents the correlations between memory performance (proportion of hits in the remote condition) and PPI connectivity scores for schema objects as indicator of the strength of the PPI.

Morris et al., 1979), which suggests that schemas are only effective as aids to memory if they are activated. This was first shown in Bransford's famous story about "washing clothes," a schema that everyone has presumably obtained, but which in the story was obscured by the manner in which the passage of prose was written. Once the theme was revealed, its effectiveness for supporting memory is dramatic. Third, in parallel with the buildup in AG, we observed that the hippocampus decreased its activity when a schema was presented. Disengagement of the hippocampus from encoding a schema object fits with the notion that schemas are stored in the neocortex and accelerate systems-level consolidation (Tse et al., 2007; Tse et al., 2008; van Kesteren et al., 2010a). It also provides a potential explanation why schema memories were found to be less specific, or less detailed, compared with memories that were not embedded in a schema.

There are subtle features of the behavioral data related to the specificity of memory. For example, the behavioral schema effect (positive difference in memory scores between the schema and no-schema conditions) was not present immediately after encoding, but emerged after consolidation. This fits with previous reports of a benefit for schema memories after a night of sleep (Tse et al., 2007; van Kesteren et al., 2013a; Durrant et al., 2015). Comparing remote and recent schema memories within participants enabled us to conclude that the schema effect reflects better retention across time for schema objects, indicating that a schema makes memories more resilient to forgetting. However, such a result could also be explained by using a schema for informed guessing after a delay, so we sought data that might help to distinguish between a consolidation and guessing interpretation. Overall, for schema and non-schema conditions, participants made more false alarms and less correct rejections to the lures that were similar to the targets relative to new lures. This has also been reported in other studies (Gutchess and Schacter, 2012; Bowman and Dennis, 2015). However, we also observed that our participants made more false alarms to similar lures of targets that might potentially have been embedded in a schema during encoding relative to lures from both non-schema conditions. This subtle detail indicates that schema memories can be less detailed and possibly more gist-based. However, the critical comparison to rule out guessing was whether the participants would falsely recognize new objects that were related to the schema but not seen during encoding; that is, they were first encountered during item recognition. It turns out they did not make more false alarms to these new schema lures compared with new no-schema lures. In fact, they made more correct rejections to the new schema lures. These findings together suggest that schemas are not used for guessing, but rather that objects related to a schema are encoded in a less specific manner, thereby giving rise to more false alarms directly after encoding.

Might the finding of less specificity for memories of schema objects be related to the reduced involvement of the hippocampus in memorizing objects from a schema? The hippocampus has circuitry well suited for discriminating between studied items and similar lures and does so through pattern separation (Bakker et al., 2008; Yassa and Stark, 2011). In our study, we found that 
targets and similar lures from a schema were more difficult to dissociate. One possibility is that a schema could act, in part, as an inhibitor of hippocampal functioning, as proposed by the SLIMM framework (van Kesteren et al., 2012). In our experiment, the AG showed functional coupling with the posterior hippocampus. More schema-related activity in AG was associated with greater deactivation of the hippocampus. In addition, a larger influence of AG on hippocampus was related to more false alarms to new schema objects, indicative that the gist but not the details of the memory were preserved. Overall, the finding of less hippocampal involvement for schema memories fits with the findings from rodents (Tse et al., 2007; Tse et al., 2008) and humans (van Kesteren et al., 2010a; van Kesteren et al., 2014) and provides more evidence for accelerated consolidation of schema memories. In addition, it provides an explanation for why schema memories seem less detailed and more gist based (Lewis and Durrant, 2011).

What is the relative role of $\mathrm{mPFC}$ and AG? To date, the partial disengagement of the MTL memory system for schema memories has been linked to the allocation of the neural correlates of schema memory to the mPFC during both encoding (van Kesteren et al., 2010a; van Kesteren et al., 2012; van Kesteren et al., 2013b; van Kesteren et al., 2014) and retrieval (van Kesteren et al., 2010b; Brod et al., 2015). We found no evidence for involvement of the mPFC during encoding of objects embedded in a schema, but the MPFC did show an interaction between study test delay and schema condition when monitored at the time of retrieval. This result confirms extensive data pointing to the involvement of the $\mathrm{mPFC}$ in retrieving remote memories (Bontempi et al., 1999; Frankland et al., 2004; Maviel et al., 2004; Takashima et al., 2006; Takehara-Nishiuchi et al., 2006; Gais et al., 2007; TakeharaNishiuchi and McNaughton, 2008; Takashima et al., 2009; Goshen et al., 2011; Bonnici et al., 2012). In contrast, the retention of schema objects for $24 \mathrm{~h}$ was related to a buildup of schema activity in the AG during encoding. The AG is high up in the hierarchy of convergence for unimodal and supramodal representations (Fernandino et al., 2016), which fits with the content of a schema. In addition, the AG is involved in processing thematic relationships (Kalénine et al., 2009), closely resembling the kind of schemas used in our experiment. This also fits with the notion that the AG is a "binding zone" that is believed to combine representations in a conceptual manner (Binder et al., 2009; Binder and Desai, 2011; Shimamura, 2011; Price et al., 2015). This binding function of the AG has recently been linked directly to recombining visual representations into schemas (Wagner et al., 2015), consistent with our findings.

Connectivity analyses using PPI revealed that object representations in LOC seemed to coactivate along with the related schema in AG. Typically, response patterns in LOC elicited by pictures of objects show categorical clustering (Eger et al., 2008). This clustering is remarkably consistent across species (Kriegeskorte et al., 2008) and reflects categorical and shape similarity (Bracci and Op de Beeck, 2016). It is likely that the AG receives "bottom-up" information when object representations that show conceptual clustering are activated. The AG then binds these related representations in a schema during encoding. During retrieval, the AG responded more to those objects that were embedded in a schema during encoding compared with nonschema objects. This likely reflects that the schema was also active during retrieval. Such retrieval-associated AG activation of a schema (consisting of related and connected object representations) leads to the expectation that the AG would similarly be connected to ventral visual representation areas such as LOC during item recognition.
The successful linking of the schema to these object representations would be beneficial to memory scores, as was observed. In addition, connectivity from AG to visual representation area such as the LOC, overlapping with those found in our object localizer, was higher for remote schema memories than for remote no-schema memories. This finding corroborates a recent study in which it was also shown that the interplay between AG and ventral visual areas was important for memory success (Kuhl and Chun, 2014). Therefore, during retrieval, schema information is used to reactivate object representations in LOC and to match the target to the previously seen object representations.

To conclude, this study provides behavioral and neuronal evidence to support the idea that schemas give rise to improved memory consolidation. The mPFC is involved in the retrieval of memories dependent on schemas. However, at least for the type of task described here, the schema information itself appears to be stored in the AG. Schema activation in AG at encoding determines whether an object is remembered successfully after $24 \mathrm{~h}$ The AG binds together schema-related object representations during encoding and uses this information again during retrieval. The simultaneous disengagement of the hippocampus from schema memory formation is further evidence for accelerated schema-associated consolidation and provides a potential explanation of why schema memories are less detailed.

\section{References}

Alvarez P, Squire LR (1994) Memory consolidation and the medial temporal lobe: a simple network model. Proc Natl Acad Sci U S A 91:7041-7045. CrossRef Medline

Bakker A, Kirwan CB, Miller M, Stark CE (2008) Pattern separation in the human hippocampal CA3 and dentate gyrus. Science 319:1640-1642. CrossRef Medline

Binder JR, Desai RH (2011) The neurobiology of semantic memory. Trends Cogn Sci 15:527-536. CrossRef Medline

Binder JR, Desai RH, Graves WW, Conant LL (2009) Where is the semantic system? A critical review and meta-analysis of 120 functional neuroimaging studies. Cereb Cortex 19:2767-2796. CrossRef Medline

Bonnici HM, Chadwick MJ, Lutti A, Hassabis D, Weiskopf N, Maguire EA (2012) Detecting representations of recent and remote autobiographical memories in vmPFC and hippocampus. J Neurosci 32:16982-16991. CrossRef Medline

Bontempi B, Laurent-Demir C, Destrade C, Jaffard R (1999) Timedependent reorganization of brain circuitry underlying long-term memory storage. Nature 400:671-675. CrossRef Medline

Bowman CR, Dennis NA (2015) The neural correlates of correctly rejecting lures during memory retrieval: the role of item relatedness. Exp Brain Res 233:1963-1975. CrossRef Medline

Boynton GM, Engel SA, Glover GH, Heeger DJ (1996) Linear systems analysis of functional magnetic resonance imaging in human V1. J Neurosci 16:4207-4221. Medline

Bracci S, Op de Beeck H (2016) Dissociations and associations between shape and category representations in the two visual pathways. J Neurosci 36:432-444. CrossRef Medline

Bransford J (1979) The role of prior knowledge. Human cognition: learning, understanding and remembering conceptual processes. Belmont, CA: Wadsworth Publishing Company.

Bransford JD, Johnson MK (1972) Contextual prerequisites for understanding: some investigations of comprehension and recall. J Verbal Learning Verbal Behav 11:717-726. CrossRef

Brod G, Lindenberger U, Werkle-Bergner M, Shing YL (2015) Differences in the neural signature of remembering schema-congruent and schemaincongruent events. Neuroimage 117:358-366. CrossRef Medline

Danker JF, Anderson JR (2010) The ghosts of brain states past: remembering reactivates the brain regions engaged during encoding. Psychol Bull 136:87-102. CrossRef Medline

Durrant SJ, Cairney SA, McDermott C, Lewis PA (2015) schemaconformant memories are preferentially consolidated during REM sleep. Neurobiol Learn Mem 122:41-50. CrossRef Medline

Eger E, Ashburner J, Haynes JD, Dolan RJ, Rees G (2008) fMRI activity 
patterns in human LOC carry information about object exemplars within category. J Cogn Neurosci 20:356-370. CrossRef Medline

Fernandino L, Binder JR, Desai RH, Pendl SL, Humphries CJ, Gross WL, Conant LL, Seidenberg MS (2016) Concept representation reflects multimodal abstraction: a framework for embodied semantics. Cereb Cortex 26:2018-2034. Medline

Frankland PW, Bontempi B (2005) The organization of recent and remote memories. Nat Rev Neurosci 6:119-130. Medline

Frankland PW, Bontempi B, Talton LE, Kaczmarek L, Silva AJ (2004) The involvement of the anterior cingulate cortex in remote contextual fear memory. Science 304:881-883. CrossRef Medline

Friston KJ, Buechel C, Fink GR, Morris J, Rolls E, Dolan RJ (1997) Psychophysiological and modulatory interactions in neuroimaging. Neuroimage 6:218-229. CrossRef Medline

Gais S, Albouy G, Boly M, Dang-Vu TT, Darsaud A, Desseilles M, Rauchs G, Schabus M, Sterpenich V, Vandewalle G, Maquet P, Peigneux P (2007) Sleep transforms the cerebral trace of declarative memories. Proc Natl Acad Sci U S A 104:18778-18783. CrossRef Medline

Ghosh VE, Gilboa A (2014) What is a memory schema? A historical perspective on current neuroscience literature. Neuropsychologia 53:104-114. CrossRef Medline

Goshen I, Brodsky M, Prakash R, Wallace J, Gradinaru V, Ramakrishnan C, Deisseroth K (2011) Dynamics of retrieval strategies for remote memories. Cell 147:678-689. CrossRef Medline

Gutchess AH, Schacter DL (2012) The neural correlates of gist-based true and false recognition. Neuroimage 59:3418-3426. CrossRef Medline

Hanson SJ, Hanson C, Halchenko Y, Matsuka T, Zaimi A (2007) Bottom-up and top-down brain functional connectivity underlying comprehension of everyday visual action. Brain Struct Funct 212:231-244. CrossRef Medline

Kalénine S, Peyrin C, Pichat C, Segebarth C, Bonthoux F, Baciu M (2009) The sensory-motor specificity of taxonomic and thematic conceptual relations: a behavioral and fMRI study. Neuroimage 44:1152-1162. CrossRef Medline

Kriegeskorte N, Mur M, Ruff DA, Kiani R, Bodurka J, Esteky H, Tanaka K, Bandettini PA (2008) Matching categorical object representations in inferior temporal cortex of man and monkey. Neuron 60:1126-1141. CrossRef Medline

Kuhl BA, Chun MM (2014) Successful remembering elicits event-specific activity patterns in lateral parietal cortex. J Neurosci 34:8051-8060. CrossRef Medline

Lee IA, Preacher KJ (2013) Calculation for the test of the difference between two dependent correlations with no variable in common [computer software]. Available from http://quantpsy.org.

Lewis PA, Durrant SJ (2011) Overlapping memory replay during sleep builds cognitive schemata. Trends Cogn Sci 15:343-351. CrossRef Medline

Malach R, Reppas JB, Benson RR, Kwong KK, Jiang H, Kennedy WA, Ledden PJ, Brady TJ, Rosen BR, Tootell RB (1995) Object-related activity revealed by functional magnetic resonance imaging in human occipital cortex. Proc Natl Acad Sci U S A 92:8135-8139. CrossRef Medline

Maviel T, Durkin TP, Menzaghi F, Bontempi B (2004) Sites of neocortical reorganization critical for remote spatial memory. Science 305:96-99. CrossRef Medline

Morris CD, Stein BS, Bransford JD (1979) Prerequisites for the utilization of knowledge in the recall of prose passages. J Exp Psychol Hum Learn Mem 5:253-261. CrossRef

Nyberg L, Habib R, McIntosh AR, Tulving E (2000) Reactivation of encoding-related brain activity during memory retrieval. Proc Natl Acad Sci U S A 97:11120-11124. CrossRef Medline

Price AR, Bonner MF, Peelle JE, Grossman M (2015) Converging evidence for the neuroanatomic basis of combinatorial semantics in the angular gyrus. J Neurosci 35:3276-3284. CrossRef Medline

Scoville WB, Milner B (1957) Loss of recent memory after bilateral hippocampal lesions. J Neurol Neurosurg Psychiatry 20:11-21. CrossRef Medline

Shimamura AP (2011) Episodic retrieval and the cortical binding of relational activity. Cogn Affect Behav Neurosci 11:277-291. CrossRef Medline

Squire LR (1986) Mechanisms of memory. Science 232:1612-1619. CrossRef Medline

Sweegers CC, Takashima A, Fernández G, Talamini LM (2014) Neural mechanisms supporting the extraction of general knowledge across episodic memories. Neuroimage 87:138-146. CrossRef Medline

Takashima A, Petersson KM, Rutters F, Tendolkar I, Jensen O, Zwarts MJ, McNaughton BL, Fernández G (2006) Declarative memory consolidation in humans: a prospective functional magnetic resonance imaging study. Proc Natl Acad Sci U S A 103:756-761. CrossRef Medline

Takashima A, Nieuwenhuis IL, Jensen O, Talamini LM, Rijpkema M, Fernández G (2009) Shift from hippocampal to neocortical centered retrieval network with consolidation. J Neurosci 29:10087-10093. CrossRef Medline

Takehara-Nishiuchi K, McNaughton BL (2008) Spontaneous changes of neocortical code for associative memory during consolidation. Science 322:960-963. CrossRef Medline

Takehara-Nishiuchi K, Nakao K, Kawahara S, Matsuki N, Kirino Y (2006) Systems consolidation requires postlearning activation of NMDA receptors in the medial prefrontal cortex in trace eyeblink conditioning. J Neurosci 26:5049-5058. CrossRef Medline

Talairach J, Tournoux P (1988) Co-planar stereotaxic atlas of the human brain. 3-D proportional system: an approach to cerebral imaging. New York: Thieme Medical Publishers.

Tse D, Langston RF, Kakeyama M, Bethus I, Spooner PA, Wood ER, Witter MP, Morris RG (2007) schemas and memory consolidation. Science 316:76-82. CrossRef Medline

Tse D, Langston RF, Bethus I, Wood ER, Witter MP, Morris RG (2008) Does assimilation into schemas involve systems or cellular consolidation? It's not just time. Neurobiol Learn Mem 89:361-365. CrossRef Medline

Tse D, Takeuchi T, Kakeyama M, Kajii Y, Okuno H, Tohyama C, Bito H, Morris RG (2011) schema-dependent gene activation and memory encoding in neocortex. Science 333:891-895. CrossRef Medline

Tulving E, Thomson DM (1973) Encoding specificity and retrieval processes in episodic memory. Psychol Rev 80:352-373. CrossRef

van Buuren M, Kroes MC, Wagner IC, Genzel L, Morris RG, Fernández G (2014) Initial investigation of the effects of an experimentally learned schema on spatial associative memory in humans. J Neurosci 34:1666216670. CrossRef Medline

van Kesteren MT, Fernández G, Norris DG, Hermans EJ (2010a) Persistent schema-dependent hippocampal-neocortical connectivity during memory encoding and postencoding rest in humans. Proc Natl Acad Sci U S A 107:7550-7555. CrossRef Medline

van Kesteren MT, Rijpkema M, Ruiter DJ, Fernández G (2010b) Retrieval of associative information congruent with prior knowledge is related to increased medial prefrontal activity and connectivity. J Neurosci 30:15888 15894. CrossRef Medline

van Kesteren MT, Ruiter DJ, Fernández G, Henson RN (2012) How schema and novelty augment memory formation. Trends Neurosci 35:211-219. CrossRef Medline

van Kesteren MT, Rijpkema M, Ruiter DJ, Fernández G (2013a) Consolidation differentially modulates schema effects on memory for items and associations. PLoS One 8:e56155. CrossRef Medline

van Kesteren MT, Beul SF, Takashima A, Henson RN, Ruiter DJ, Fernández G (2013b) Differential roles for medial prefrontal and medial temporal cortices in schema-dependent encoding: from congruent to incongruent. Neuropsychologia 51:2352-2359. CrossRef Medline

van Kesteren MT, Rijpkema M, Ruiter DJ, Morris RG, Fernández G (2014) Building on prior knowledge: schema-dependent encoding processes relate to academic performance. J Cogn Neurosci 26:2250-2261. CrossRef Medline

Wagner IC, van Buuren M, Kroes MC, Gutteling TP, van der Linden M, Morris RG, Fernández G (2015) schematic memory components converge within angular gyrus during retrieval. Elife 4:e09668. CrossRef Medline

Wang SH, Morris RG (2010) Hippocampal-neocortical interactions in memory formation, consolidation, and reconsolidation. Annu Rev Psychol 61:49- 79, C41-44. CrossRef Medline

Winocur G, Moscovitch M, Bontempi B (2010) Memory formation and long-term retention in humans and animals: convergence towards a transformation account of hippocampal-neocortical interactions. Neuropsychologia 48:2339-2356. CrossRef Medline

Yassa MA, Stark CE (2011) Pattern separation in the hippocampus. Trends Neurosci 34:515-525. CrossRef Medline 